\title{
X-RAY OPTICS AND PRECISION REQUIREMENTS FOR RESIDUAL STRESS ANALYSES WITH HIGH SPATIAL RESOLUTION
}

\author{
Bernd Eigenmann \\ X-Ray and Materials Laboratory Eigenmann \\ Felsenweg 6, 91220 Schnaittach-Hormersdorf, Germany \\ e-mail: bernd.eigenmann@eigenspannungen.de
}

Keywords: glass capillary optics, spatial resolution, alignment precision, curved surfaces

\begin{abstract}
In recent years, the demand for high spatial resolution in X-ray residual stress analysis has drastically increased. The locations of interest are frequently small foot radii of teeth of gears. Also the inner surface of holes or hollow cylinders in general with small diameter must be investigated after opening the cylindrical cavities. In resolving such measuring problems, significant progress has been made in reproducibly manufacturing and applying glass capillary X-ray optics. With focusing elliptical polycapillaries and conventional laboratory X-ray sources, spot sizes of few $10 \mu \mathrm{m}$ can be realized at sufficiently intensities for residual stress analyses.

However, glass capillary optics require refined alignment strategies which are completely different from those for conventional beam shaping optics. Moreover, the small spot sizes cannot be aligned and positioned on the sample surface by eye. Microscopy fixtures are required. Finally, measurements in small radii result in high precision requirements for the diffractometers as well as for the sample positioning in axes and directions which are significantly less relevant when measuring on plane surfaces.

The specific requirements resulting from residual stress analyses with high spatial resolution using glass capillaries and small spot sizes at curved surfaces are described and discussed.
\end{abstract}

\section{Introduction}

The increasing demand for X-ray residual stress analyses with improved spatial resolution has led to developments of X-ray optics which can be used at conventional laboratory X-ray sources an which produce beam cross sections with down to about $40 \mu \mathrm{m}$ at the sample surface while still yielding intensities sufficiently high for diffraction experiments in general and X-ray residual stress analyses with still reasonable measuring times. The use of glass capillaries requires more alignment effort than that of pinhole and slit optics. Nevertheless, the advantage if used for improved spatial resolutions at plain surfaces is significant. If used at curved surfaces, the alignment and the precision of the machine can become a critical issue. In the following, the principles of glass capillary optics are briefly explained. The relevant systematical geometrical error sources of X-ray diffractometers are described. The sensitivity of X-ray residual stress analyses to alignment errors is discussed for different diffraction conditions and for plane and curved surfaces.

\section{X-Ray Residual Stress Analysis}

$\mathrm{X}$-ray residual stress analysis is based on the diffraction of monochromatic X-rays of wavelength $\lambda$ by the crystal lattice of the generally polycrystalline material under investigation. The measured Bragg angles $\theta$ are related to the lattice plain spacings $d$ according to Bragg's equation

$$
\lambda=2 d \sin \theta .
$$


Residual stresses and strains result in lattice strains which can be derived from the shift of Bragg angles resulting from them as

$$
\varepsilon=\frac{d-d_{0}}{d_{0}}=-\left(\theta-\theta_{0}\right) \cot \theta_{0}
$$

with the index 0 denoting the values related to the stress-free crystal lattice. A suitable sample-fixed coordinate system is chosen as reference system for residual stress analyses (s. scheme of sample and diffractometer in fig. 1). Lattice strains are determined in different directions with respect to that coordinate system, and residual stresses are calculated from them using special X-ray elastic constants which account for the influence of the elastic anisotropy of the crystal lattice. Applying the most widely used $\sin ^{2} \psi$-method [1], an individual residual stress component parallel to the surface in direction $\varphi$ with respect to the $\mathrm{x}_{1}$-axis is calculated from lattice strains $\varepsilon_{\varphi, \psi}$ measured in the azimuth $\varphi$ at several distance angles $\psi$ with respect to the $\mathrm{x}_{3}$-axis normal to the surface. In fig. 1 , the measuring direction is identical with the $\mathrm{x}_{1}$-axis, and $\varphi=0^{\circ}$. The equation

$$
\varepsilon_{\varphi, \psi}=\frac{v^{\{h k l\}}+1}{E^{\{h k l\}}} \sigma_{\varphi} \sin ^{2} \psi-\frac{v^{\{h k l\}}}{E^{\{h k l\}}}\left(\sigma_{1}+\sigma_{2}\right)
$$

allows the calculation of the stress value $\sigma_{\varphi}$ from the slope of a straight line resulting if the lattice strains $\varepsilon_{\varphi, \psi}$ are plotted versus $\sin ^{2} \psi$. The X-ray values of Young's modulus $E^{[\mathrm{hkl}\}}$ and of Poisson's ratio $v^{\{h k 1\}}$ are specific for the selected lattice planes $\{h \mathrm{hl}\}$. For more details, see [2]. For the stress analyses, the samples are positioned in the center of a diffractometer as schematically shown in fig. 1 for a $\Psi$-diffractometer [3]. Ideally, it is assumed that the setup is free of geometrical errors. This means that in the ideal case, the center of the X-ray beam, the sample surface, and the $\varphi-, \psi-, \theta-$, and $2 \theta-$ axes ideally meet in the theoretical center of the diffractometer. However, in some cases the influences of practically present geometrical errors become relevant. Therefore, the most important geometrical errors are described, and their influences on residual stress measurements are discussed in the following.

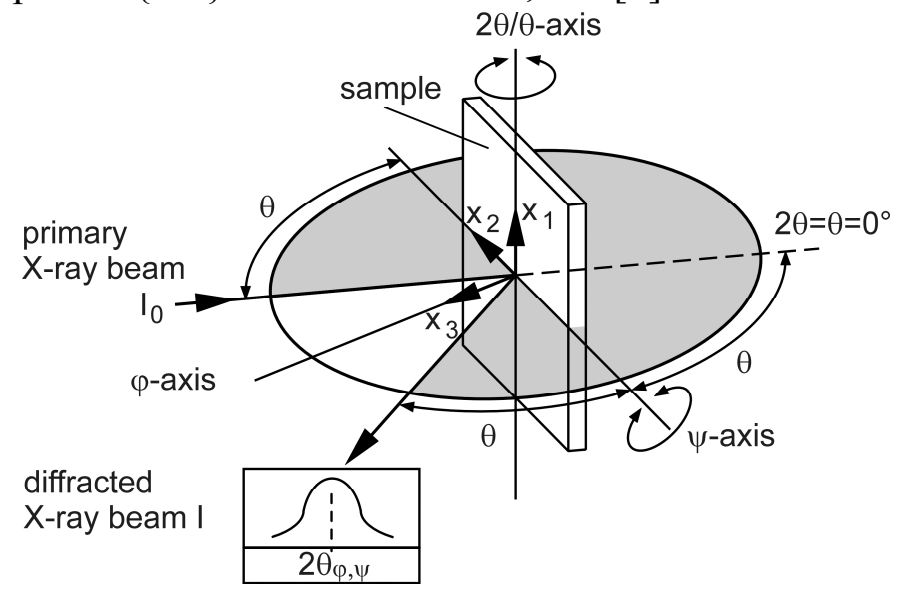

Figure 1: Geometrical principles of a $\Psi$-diffractometer with sample in position $\varphi=0^{\circ}, \psi=0^{\circ}$ and sample-fixed coordinate system.

\section{Geometrical Errors Influencing Residual Stress Analyses}

The errors discussed in the following only consider mechanical precision and alignment aspects. They do not include counting statistics of X-rays nor the evaluation procedure of the diffraction peak positions. It should also be mentioned that the use of small X-ray spots can lead to a large scatter of measuring results due to grain size of the material and due to material inhomogeneities. Additional care can be required to achieve useful results. This issue is, however, not subject of this paper and not treated in the following. For practical reasons, three portions of the total mechanical error are considered separately.

"Non-Systematical" Part of the Error Sphere of the Mechanical Setup. This part of the total error sphere is determined by the design and manufacturing precision of the machine. It contains distortions due to temperature changes and due to bearing constraints as well as elastic deformation under load due to lacking stiffness. The movement of the ideal machine center within this error 
sphere is typically not following simple circular or elliptic traces but can appear quite chaotic. Whereas most of the systematical geometrical errors are sufficiently constant during a stress measurement, this portion of the total error cannot be controlled with sufficient reliability. Good commercially available 3 to 7 axis machines are specified with error sphere radii near $30 \mu \mathrm{m}$. Many machines, however, are not precisely specified or much worse.

Systematical Alignment Errors of Primary Optics. The elements of the primary optics are frequently exchanged depending on the measurement requirements. They are typically aligned with machine-specific alignment procedures using fluorescent screens and stress-free powder samples. Small remaining errors are systematical and can be identified and - if needed - subsequently corrected using reference measurements on the stress-free powder samples as external standards.

Pinhole or Slit Optics. If conventional pinhole or slit optics are used to shape the cross section of the primary beam, the alignment simply requires the positioning of the center of the primary slit on the line connecting the center of the diffractometer with the center of the focus of the X-ray tube. To center the beam to the diffractometer axes (typically $\theta$ - and $\psi$-axis), one translatoric movement of the primary slit is required per axis. The arrangement can be seen at the top of fig. 2 .

Glass Capillary Optics. Glass capillary optics are increasingly used since they deliver significantly higher intensity than pinhole optics, especially if small spot sizes are used. Some principles are described in [4]. For high intensities and spot sizes of several $100 \mu \mathrm{m}$ to about $2 \mathrm{~mm}$, in most cases cylindrical mono- or polycapillaries are used. The principle is illustrated in the center part of fig. 2. The principle is the total reflection of the X-radiation at the inner wall of the glass capillary. Since the angle of total reflection is, depending on the glass and the radiation wavelength, $0.25^{\circ}$ to $0.35^{\circ}$, the divergence $2 \theta_{\mathrm{c}}$ of the incoming and exit beam is in the range of about $0.5^{\circ}$ to $0.7^{\circ}$, i.e. the beam divergence is larger than for most pinhole or slit optics. As a consequence of the typically large distance $\mathrm{x}_{0}$ between tube focus and capillary entrance end, the entrance cone of cylindrical capillaries sees all or most of the focus of the X-ray tube. With such a setup, even with small capillary diameters $D_{c}$, it is impossible to realize very small spot sizes at the sample surface since for

pinhole optics
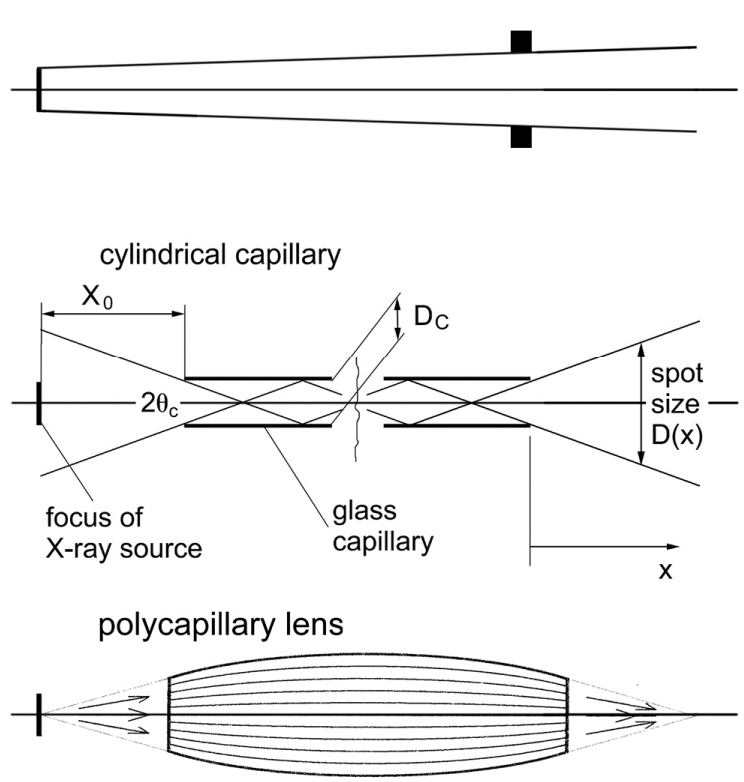

Figure 2: Principles of primary X-ray optics: Pinhole optics (top), cylindrical glass capillary (center), and focusing glass polycapillary lens (bottom). every millimeter distance $\mathrm{x}$ between the end of the capillary and the sample surface, the beam diameter $\mathrm{D}(\mathrm{x})$ increases by about $10 \mu \mathrm{m}$.

For very small spot sizes, elliptical poly-capillary lens are used as illustrated in the lower part of fig. 2. The beam divergence angle is typically in the range of $1^{\circ}$. Spot sizes on the sample in the exit focus of the capillary can be reduced down to $40 \mu \mathrm{m}$ to $60 \mu \mathrm{m}$. The entrance focus of the capillary is placed in the center of the focus of the X-ray tube.

The alignment of capillary optics is more delicate than that of pinhole or slit optics. The center line of the capillary must be aligned with the line between center of tube focus and center of the diffractometer using fluorescent screens and stressfree powder standards. This alignment requires one translation and one rotation of the capillary per axis to be aligned (typically $\theta$ - and $\psi$-axis). The centering with respect to the tube focus and the alignment must be done carefully. Errors can cause asymmetrical intensity and wavelength gradients across the exit beam which can have awkward effects on the measured results. 
Systematical Mechanical Alignment Errors. This are small remaining errors after mechanically assembling the machines, if two or more axes do not perfectly intersect in the diffractometer center or if the sample surface is not perfectly aligned in the diffractometer center.

As shown in [2], all small systematical errors in the diffractometer plane can be described as illustrated in fig. 3 by a displacement of the primary beam $\Delta \mathrm{P}$, by a displacement of the secondary slit $\Delta \mathrm{S}$, and by a displacement of the sample $\Delta \mathrm{H}$. The resulting shift

$$
\Delta 2 \theta^{\{h k l\}}=2 \theta_{\text {ideal }}^{\{h k l\}}-2 \theta_{\text {measured }}^{\{h k l\}}
$$

varies linearly with $\cos \theta$. If, as illustrated in fig. 4 for $\mathrm{W}$-powder and Fe K $\alpha$-radiation, $\Delta 2 \theta^{\{\mathrm{hkl}\}}$ is determined for different diffraction profiles, a linear correction factor

$$
\Delta 2 \theta=K_{\theta} \cos \theta+K
$$

can be calculated which can be used to correct the measured $2 \theta$-values according to eq. 4 .

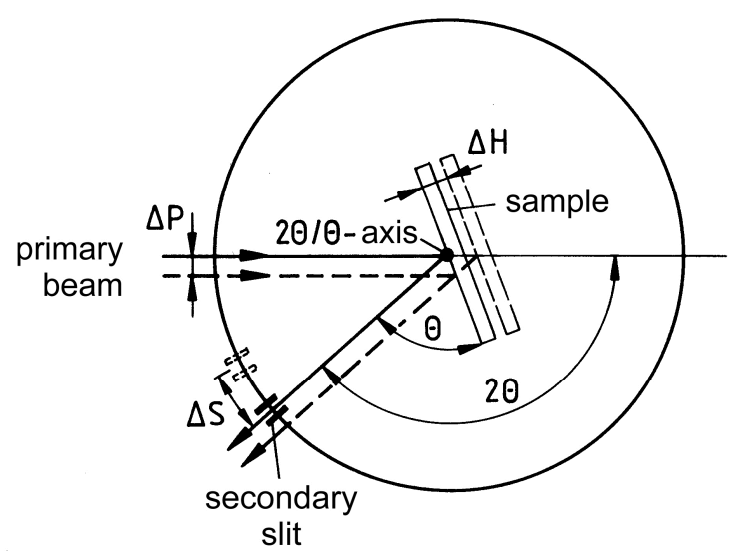

Figure 3: Possible geometrical errors in the diffractometer plane: displacement of primary beam $\Delta \mathrm{P}$, of the sample $\Delta \mathrm{H}$, and of the secondary slit $\Delta \mathrm{S}$.

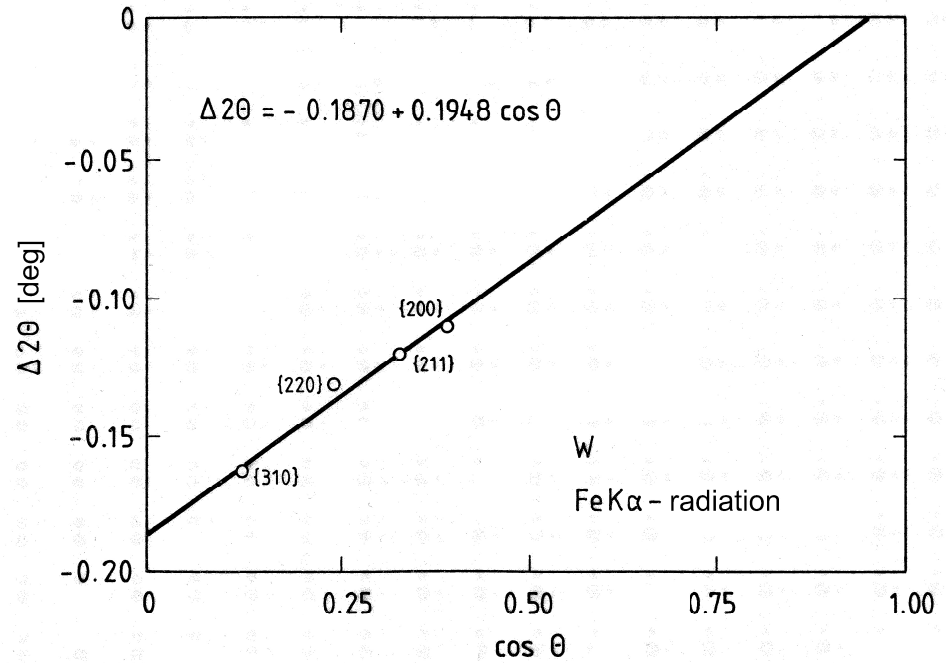

Figure 4: Correction function for geometrical errors in the diffractometer plane, determined exemplarily with Fe Ko-radiation on W-powder.

\section{Sensitivity of Residual Stress Analyses to Geometrical Errors}

General Aspects. Resulting from the diffraction on the crystal lattice, the $\cot \theta$ in eq. 2 leads to an increasing effect of geometrical beam displacements on resulting strains and stresses with lower $2 \theta$. This is well known and the reason why residual stress analyses are preferably done in the high angle backscattering range. Due to the $\cot \theta$, residual stress analyses, as an example, with $\mathrm{Cr}$ K $\alpha$-radiation on $\{211\}$-lattices planes of steel at $2 \theta \approx 156.4^{\circ}$ are by a factor of 7 less sensitive to beam displacements than it would be on $\{110\}$-lattice planes at $2 \theta \approx 68.8^{\circ}$.

Moreover, the diffractometer radius plays an important role. At a radius of $\mathrm{R}=150 \mathrm{~mm}$, the results are about twice as sensitive to geometrical beam displacements as at a Radius of $\mathrm{R}=290 \mathrm{~mm}$ (where $1^{\circ}$ in $2 \theta$ corresponds to $5 \mathrm{~mm}$ on the diffractometer circle).

Plane Sample Surfaces. In case of plane sample surfaces, the well-known sensitivities to errors as described above are valid. The influences can be easily calculated and quantified. The systematical mechanical errors of a $\Psi$-diffractometer follow a $2 \cos \theta / \cos \psi$-rule which implies that, as an example, residual stress analyses with $\mathrm{Cr} \mathrm{K} \alpha$-radiation on $\{211\}$-lattices planes of steel at $2 \theta \approx 156.4^{\circ}$ are by a factor of 4 less sensitive to mechanical errors than it would be on $\{110\}$-lattice planes at $2 \theta \approx 68.8^{\circ}$. Together with the above-described diffraction effect, this makes residual stress analyses, again as an example, on $\{211\}$-lattices planes of steel at $2 \theta \approx 156.4^{\circ}$ almost by a factor of 30 less sensitive to errors than it would be on $\{110\}$-lattice planes at $2 \theta \approx 68.8^{\circ}$. This is illustrated in fig. 5 . 


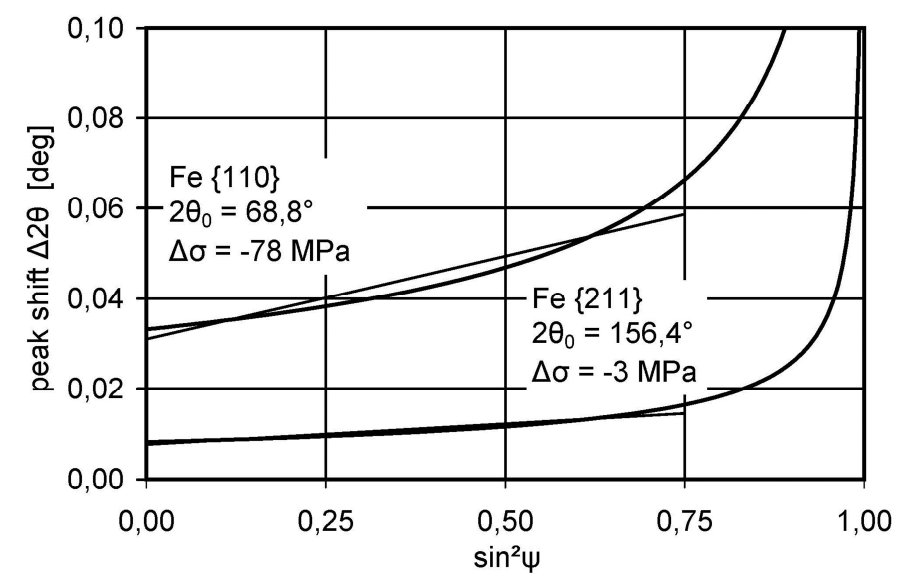

Figure 5: Calculated peak shifts $\Delta 2 \theta$ resulting from a sample displacement $\Delta \mathrm{x}_{3}=0.1 \mathrm{~mm}$ for measuring conditions with $\mathrm{Cr} \mathrm{K \alpha} \alpha$-radiation on Fe $\{110\}$ - and $\{211\}$-planes and resulting stress errors $\Delta \sigma$ for linear evaluation up to $\sin ^{2} \psi=0.75$.
For the measuring conditions above on $\{211\}$ - and $\{110\}$-lattice planes of steel, the peak shift $\Delta 2 \theta$ resulting from an assumed sample displacement $\Delta \mathrm{x}_{3}=0.1 \mathrm{~mm}$ is calculated and plotted vs. $\sin ^{2} \psi$. As expected, the lines show the above-mentioned higher sensitivity to the geometrical error in case of $\{110\}$-lattice planes at $2 \theta \approx 68.8^{\circ}$. It also shows that the peak shift increases drastically with increasing $\psi$. It would become infinite at $\psi=90^{\circ}$. If measuring up to $\psi= \pm 60^{\circ}$ $\left(\sin ^{2} \psi \leq 0.75\right)$ is considered, the peak shift due to sample displacement appears to be almost linear. It can be evaluated with the parameters valid for steel, and the sample displacement would create an error of residual stress of $\Delta \sigma=-3 \mathrm{MPa}$ in case of $\{211\}-$ lattices planes of steel at $2 \theta \approx 156.4^{\circ}$ and an

error of $\Delta \sigma=-78 \mathrm{MPa}$ in case of $\{110\}$-lattice planes at $2 \theta \approx 68.8^{\circ}$ with a diffractometer radius of $\mathrm{R}=290 \mathrm{~mm}$. With a diffractometer radius of $150 \mathrm{~mm}$, the errors $\Delta \sigma$ would be approximately doubled. The error estimate proves that the standard measuring conditions on steel are quite robust in case of plane sample surfaces.

Curved Sample Surfaces. It is commonly recommended if curved surfaces are investigated to keep the measuring spot on the sample smaller than $1 / 5$ of the curvature radius. This is valid for quite robust measuring conditions comparable to those on steel $\{211\}$-lattice planes with $\mathrm{Cr} \mathrm{K} \alpha$-radiation with $\psi$ limited to $\pm 45^{\circ}$. If the $\psi$-range is increased and/or diffraction peaks at lower $2 \theta$-values are investigated, the spot size should be limited rather to $1 / 10$ of the surface curvature radius. The consideration is also only valid if the sample curvature radius and the X-ray spot size on the sample are much larger than the error sphere and alignment errors of the diffractometer. If recent advances in reducing spot sizes using focusing polycapillaries are considered to measure residual stresses in small radii, this is no more the case, and additional care has to be taken. Since side effects like possible influences of the movement of the diffractometer center within the error sphere of the machine and a partially shaded area around the full X-ray beam make it difficult to calculate effects of errors on the measured residual stresses, the sensitivity analysis was done experimentally. Again the typically robust standard conditions of residual stress analyses with $\mathrm{Cr} \mathrm{K} \alpha$-radiation on $\{211\}$-lattice planes of steel are considered. A primary beam focused to a diameter of $60 \mu \mathrm{m}$ at the surface of the sample is used. The sample is a cylinder with a shoulder, the diameters being $10 \mathrm{~mm}$ and $20 \mathrm{~mm}$. The radius between the cylinder and the shoulder is 5 times the beam diameter, i.e. $300 \mu \mathrm{m}$. Residu-

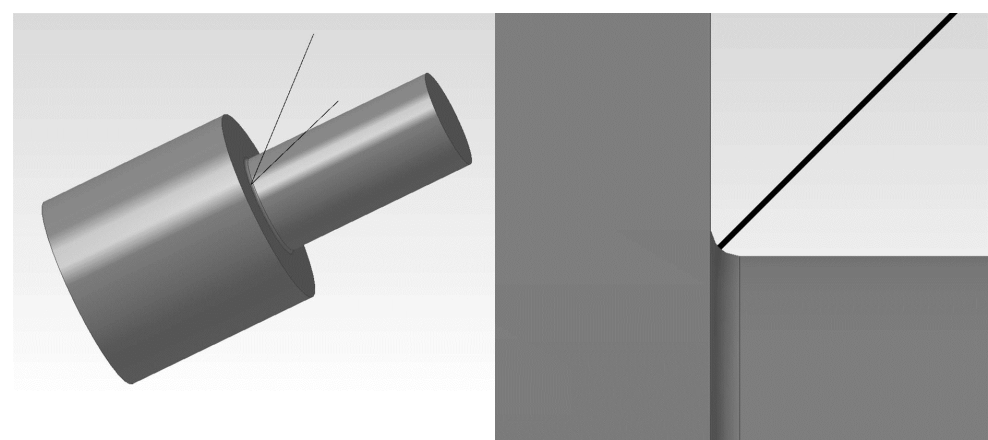

Figure 6: Geometrical conditions when measuring with a $60 \mu \mathrm{m}$ beam at the $45^{\circ}$ position of a $300 \mu \mathrm{m}$ radius with $2 \theta=156.4^{\circ}$. al stresses should be measured in the $45^{\circ}$ position of the radius in longitudinal direction of the sample. The geometrical situation with sample and $\mathrm{X}$ ray beam is illustrated in fig. 6 . As a consequence of the $90^{\circ}$ shoulder of the sample, the $\psi$-range was limited to $\pm 45^{\circ}$. The error sphere of the diffractometer used was determined using a microscope camera. The radius was $\mathrm{R}=10 \mu \mathrm{m}$. The sample was aligned to the center of the X-ray beam also using 


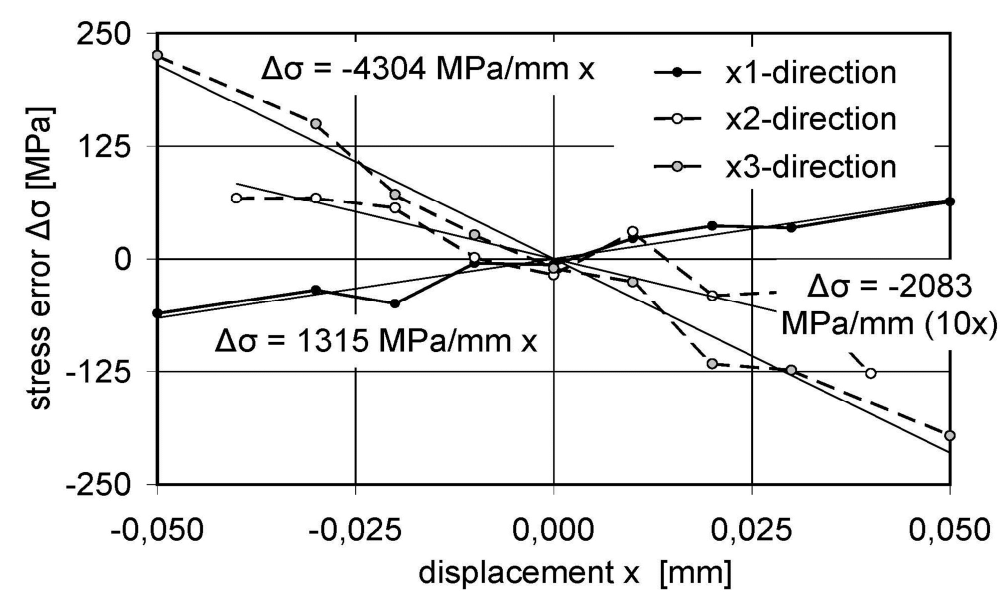

Figure 7: Stress errors $\Delta \sigma$ resulting from sample displacements in $\mathrm{x}_{1^{-}}, \mathrm{x}_{2^{-}}$, and $\mathrm{x}_{3}$-direction when using a $60 \mu \mathrm{m}$ beam in a $300 \mu \mathrm{m}$ radius at $2 \theta=156.4^{\circ}$ (the scale of the displacement in $\mathrm{x}_{2}$-direction is divided by 10 ). a microscope camera. The sensitivity analysis to sample displacement was done by shifting the sample to defined displacements along the $\mathrm{x}_{1^{-}}, \mathrm{x}_{2^{-}}$, and $\mathrm{x}_{3}$-axis. The resulting errors in residual stresses $\Delta \sigma$ are determined and displayed in fig. 7 vs. the displacement. Obviously, a sample displacement in $\mathrm{x}_{1}$-direction is not very critical and creates an error of about $1.3 \mathrm{MPa} / \mu \mathrm{m}$. The least critical direction is the $\mathrm{x}_{2}$-direction (in circumferential direction along the baseline of the shoulder radius). It causes an error of $-0.2 \mathrm{MPa} / \mu \mathrm{m}$. The most critical displacement appears to be in $\mathrm{x}_{3}$-direction perpendicular to the

surface. It causes an error of $-4.3 \mathrm{MPa} / \mu \mathrm{m}$. This also implies that already an error sphere radius of the diffractometer of $\mathrm{R}=10 \mu \mathrm{m}$ could possibly create an error of $\pm 40 \mathrm{MPa}$. An error sphere radius of $\mathrm{R}=30 \mu \mathrm{m}$ could possibly create an error of $\pm 130 \mathrm{MPa}$ which is no more acceptable. These findings prove that the precision of the machine as well as of the alignment of optics and sample is an absolutely crucial point for residual stress measurements with small spot sizes at curved surfaces with small radii.

\section{Conclusions}

It has been shown that the use of glass capillaries for X-ray residual stress analyses is advantageous in order to provide sufficient intensity even with comparably small spot sizes. However, cylindrical capillaries are not suitable for really small spot sizes. Due to the comparably large beam divergence, it is not efficient to try to achieve spot sizes with diameters significantly smaller than about $1 \mathrm{~mm}$. Spot sizes down to about $40 \mu \mathrm{m}$ to $60 \mu \mathrm{m}$ and still sufficient X-ray intensities can be achieved using focusing polycapillaries.

The sensitivity of X-ray residual stress analyses to measuring conditions and alignment errors of the diffractometers has been discussed. For plane sample surfaces, it has been shown that the alignment is comparably uncritical. The well-known influences of the measuring conditions were demonstrated. It was proved that measuring in the high angle backscattering region is by at least a factor of 30 less sensitive to alignment errors than measuring in the low angle forward scattering region.

In case of curved surfaces, it has been shown that residual stress measurements become extremely sensitive to alignment errors if the spot size reaches $1 / 5$ of the curvature radius of the surface. It has been shown that the sample displacement normal to the surface becomes most critical. It can be concluded from the findings that for measurements in such small radii, the geometrical error sphere of the diffractometer must be significantly smaller than the X-ray spot size used. The presented results imply that it should be smaller than or equal to about $1 / 6$ of the spot size.

\section{References}

[1] E. Macherauch, P. Müller, Das $\sin ^{2} \psi$-Verfahren der röntgenographischen Spannungsmessung, Z. angew. Physik 13 (1961) 305-312.

[2] B. Eigenmann, E. Macherauch, Röntgenographische Untersuchung von Spannungszuständen in Werkstoffen, Mat.-wiss. u. Werkstofftech., Teil I: 26 (1995) 148-160, Teil II: 26 (1995) 199-216, Teil III: 27 (1996) 426-437, Teil IV: 27 (1996) 491-501.

[3] U. Wolfstieg, Das $\Psi$-Diffraktometer, HTM 31 (1976) 19-22.

[4] B. Eigenmann, N. Langhoff, A. Bjeoumikhov, A. Haase, R. Stabenow, Advantageous Use of Glass Capillaries as Primary Optics for X - Ray Residual Stress Analyses and a Novel Concept for Micro Diffraction Stress Analysis, Mat. Sci. Forum 404-407 (2002) 303-308. 